\title{
High-sensitivity troponin assays for the diagnosis of AMI-sex-specific differences?
}

$\mathrm{T}$ he identification of highly sensitive biomarkers for the timely diagnosis of acute myocardial infarction (AMI) in patients admitted to the emergency department is of critical importance. Two studies published in the BMJ described the efficacy of high-sensitivity troponin assays for the diagnosis of AMI.

In a meta-analysis, Zhelev et al. report that a single baseline measurement using a high-sensitivity troponin $\mathrm{T}$ assay could rule out AMI if low cut-off values $(<5 \mathrm{ng} / \mathrm{l})$ are used. The investigators searched for diagnostic test accuracy studies published between January 2006 and December 2013. Studies describing the accuracy of the Elecsys Troponin T high-sensitive assay (Roche Diagnostics, IN, USA) for diagnosing AMI in emergency room patients with suspected acute coronary syndrome (ACS) were included.

In total, 23 studies, which included 9,428 patients, were included in the analysis. Most patients presented to the emergency department within $12 \mathrm{~h}$ of symptom onset. The performance of high-sensitivity troponin $\mathrm{T}$ in ruling out AMI at the manufacturer's recommended cut-off value of $14 \mathrm{ng} / \mathrm{l}$ was described in 20 articles. Six articles reported the results for $3 \mathrm{ng} / \mathrm{l}$ and $5 \mathrm{ng} / \mathrm{l}$ cut-off values at presentation; the data for these two cut-off points were pooled. At $14 \mathrm{ng} / \mathrm{l}$, sensitivity was $89.5 \%$ (95\% CI 86.3-92.1\%) and specificity was $77.1 \%$ (95\% CI 68.7$83.7 \%)$. At $3-5 \mathrm{ng} / \mathrm{l}$, sensitivity was $97.4 \%$ (95\% CI 94.9-98.7\%) and specificity was 42.4\% (95\% CI 31.2-54.5\%).

According to Zhivko Zhelev, an investigator in this meta-analysis, these findings demonstrate that using the " $14 \mathrm{ng} / \mathrm{l}$ cut-off value may lack the necessary diagnostic sensitivity to reliably rule out AMI in presentation samples; using lower cut-off values ( $5 \mathrm{ng} / \mathrm{l}$ or less), however, may allow reliable exclusion of AMI in most of the patients at their presentation to [the emergency room].”
In 100 consecutive patients presenting with symptoms suggestive of ACS who are tested using this assay, 21 will eventually be diagnosed with AMI. Using the $14 \mathrm{ng} / \mathrm{l}$ cut-off, this diagnosis will be missed in 2 patients (95\% CI 2-3) and 18 patients will be wrongly diagnosed with AMI (95\% CI 13-25). However, using the 3-5 ng/l cut-off value, $<1$ patient with AMI will be missed (95\% CI 0-1), and 46 patients will be incorrectly diagnosed (95\% CI 36-54).

Zhelev cautions against using the assay in patients who present less than $3 \mathrm{~h}$ after symptom onset, as "the sensitivity of the test may be lower, which means that higher number of patients with MI will be missed." Furthermore, "the total imprecision of the test at lower threshold values might be higher than the recommended coefficient of variation of $<10 \%$," which means that the assay's performance might be less reliable.

Although high-sensitive troponin assays have proven to be effective in ruling out AMI in emergency room patients, some investigators suggest that the test performs differently between males and females. In a separate study, Shah and colleagues sought to determine the diagnostic value of the ARCHITECT STAT high-sensitivity troponin-I assay (Abbott Laboratories, IL, USA) and evaluated the sex-specific diagnostic thresholds in men and women with suspected ACS.

In total, 1,126 consecutive patients with suspected ACS ( $46 \%$ of whom were women) were included in the study. Sex-specific diagnostic thresholds (men $34 \mathrm{ng} / \mathrm{l}$, women $16 \mathrm{ng} / \mathrm{l}$ ) were set for the high-sensitive assay, and compared with the contemporary troponin I assay (50 ng/l, single threshold).

Using the contemporary assay, $11 \%$ of women and $19 \%$ of men were diagnosed with AMI. Using the high-sensitive assay, AMI diagnosis increased to $16 \%$ and $22 \%$ of women using the generic $26 \mathrm{ng} / \mathrm{l}$ and the sex-specific $16 \mathrm{ng} / \mathrm{l}$ threshold,

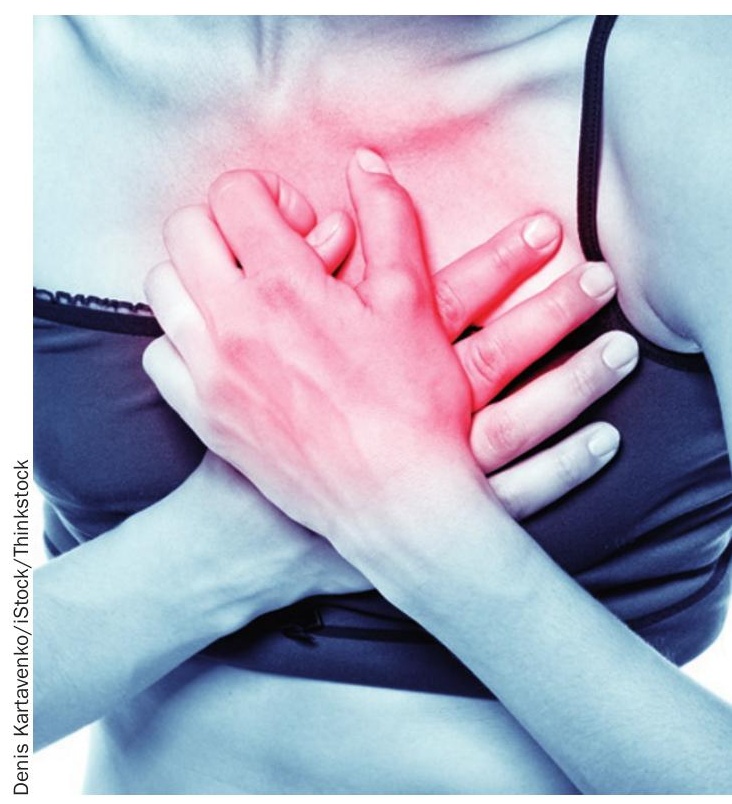

respectively. By contrast, the diagnosis of AMI in men increased only slightly to $23 \%$ and $21 \%$ using the generic $26 \mathrm{ng} / \mathrm{l}$ and the sex-specific $34 \mathrm{ng} / \mathrm{l}$ threshold, respectively.

According to Nick Mills, an investigator in this study, such sex-specific assessments are important because "currently only 1 in 10 women with chest pains will be diagnosed with AMI compared to 1 in 5 men ... the use of sex-specific thresholds will restore some balance with a similar proportion of men and women diagnosed". The investigators are currently performing a trial that will determine "whether use of this assay and sex-specific thresholds will improve survival in 26,000 patients with suspected ACS."

Karina Huynh

\footnotetext{
Original articles Zhelev, Z. et al. Diagnostic accuracy of single baseline measurement of Elecsys Troponin T highsensitive assay for diagnosis of acute myocardial infarction in emergency department: systematic review and metaanalysis. BMJ 350, h15 (2015) | Shah, A. S. V. et al. High sensitivity cardiac troponin and the under-diagnosis of myocardial infarction in women: prospective cohort study. BMJ 350, g7873 (2015)
} 